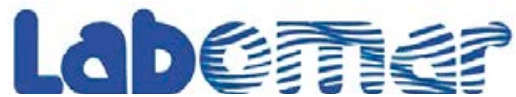

Arquivos de Ciências do Mar

\section{EFEITO DAS PERDAS DE COBERTURA DE PRADOS DE ANGIOPERMAS MARINHAS SOBRE A MACROFAUNA BENTÔNICA}

\author{
Effect of the losses of coverage of seagrass \\ meadows on the benthic macrofauna
}

\author{
Laura Costa Laranjeira ${ }^{1}$, Kcrishna Vilanova de Souza Barros ${ }^{2}$, \\ Mônica Lucia Botter-Carvalho ${ }^{3}$, Karine Matos Magalhães ${ }^{4,}{ }^{*}$
}

\begin{abstract}
${ }^{1}$ Bacharela em Ciências Biológicas, aluna do Laboratório de Ecossistemas Aquáticos da Universidade Federal Rural de Pernambuco - LEAqua/UFRPE, Rua Dom Manoel de Medeiros, S/N, Dois Irmãos, Recife-PE, CEP 52.171-900;

${ }^{2}$ Doutora em Ciências Marinhas Tropicais, em estágio pós-doutoral no Programa de Pós-Graduação em Ciências Marinhas Tropicais e no Laboratório de Zoobentos do Instituto de Ciências do Mar, Universidade Federal do Ceará Labomar/UFC, Av. da Abolição, 3207, Meireles, Fortaleza-CE, CEP 60.165-081;

${ }^{3}$ Coordenadora do Laboratório de Ecologia do Bentos Costeiro, Universidade Federal Rural de Pernambuco - LEBENC/ UFRPE, Rua Dom Manoel de Medeiros, S/N, Dois Irmãos, Recife-PE, CEP 52.171-900;

${ }^{4}$ Coordenadora do Laboratório de Ecossistemas Aquáticos, Universidade Federal Rural de Pernambuco - LEAqua/ UFRPE, Rua Dom Manoel de Medeiros, S/N, Dois Irmãos, Recife-PE, CEP 52.171-900;

*E-mail para correspondência: karinematosmagalhaes@gmail.com
\end{abstract}

\section{RESUMO}

Para avaliar o efeito de perdas recentes de áreas de pradarias marinhas sobre a macrofauna bentônica associada, foram comparadas as assembleias associadas às áreas vegetadas e áreas com perda recente de cobertura vegetal, na praia de Catuama, litoral norte de Pernambuco, Brasil. Em maio de 2010, foram coletadas 10 amostras dentro do prado de Halodule wrightii e 10 amostras em áreas não mais ocupadas pela pradaria (não-vegetadas), com auxílio de um tubo coletor de PVC, para análises das plantas e da fauna capturada em malha de $0,5 \mathrm{~mm}$. A macrofauna bentônica foi composta por 2566 espécimes, sendo 2493 em áreas vegetadas e 73 em áreas sem vegetação. Apenas anelídeos e moluscos foram encontrados em ambos os ambientes, mas a abundância total $(H=15,310 ; p<0,001)$ e o número de táxons $(\mathrm{H}=13,740 ; \mathrm{p}<0,001)$ foram significativamente maiores nas áreas vegetadas. Assim, o estudo demonstrou que as áreas não vegetadas, onde ocorreu perda de cobertura vegetal recente, foram significativamente menos habitadas, reforçando a necessidade de conservação destes prados e, consequentemente, a manutenção de seus serviços ecossistêmicos, como a fauna associada.

Palavras-chave: Bentos, Ecossistema fital, capim-agulha

Recebido em: $14 / 05 / 2018$

Aprovado em: 07/08/2018

Publicado online em: 25/03/2019 


\section{ABSTRACT}

In order to evaluate the effect of recent losses of seagrass areas among the associated benthic macrofauna, the faunistic assemblages associated with the vegetated areas and areas with recent losses of vegetal coverage were evaluated in Catuama Beach, northern coast of Pernambuco, Brazil. In May 2010, ten samples were collected within the Halodule wrightii meadow and other ten samples in areas no more occupied by the meadow (non-vegetated), with the help of a PVC collection tube, for analyses of the plants and the fauna captured in a $0.5 \mathrm{~mm}$ mesh. The macrofauna was composed by 2566 specimens, being 2493 in vegetated areas and 73 in areas without vegetation. Only annelids and molluscs were found in both environments, but the total abundance $(H=15.310$; $p<0.001)$ and the number of taxa $(H=13.740 ; p<0.001)$ were significantly higher in the vegetated areas. Thus, the study demonstrated that the non-vegetated areas, where a recent loss of vegetal coverage occurred, were significantly less inhabited, reinforcing the need of conservation of these meadows and, consequently, the maintenance of their services for the ecosystem as the associated fauna.

Key words: Benthos, phytal ecosystem, shoal grass.

\section{INTRODUÇÃO}

De um modo geral, todos os principais grupos de invertebrados marinhos estão associados a prados de angiospermas marinhas submersas (Barnes, 2017), com diferentes padrões na abundância e diversidade das assembleias faunísticas bentônicas, entre áreas vegetadas e não-vegetadas adjacentes (Barnes \& Barnes, 2012; Do et al., 2011; Edgar, 1990; Nakaoka, 2005; Orth et al., 1984; Terrados \& Borum, 2004) e curtas faixas de transição, onde a fauna se modifica em apenas 0,1 m, a partir da borda da pradaria (Barnes, 2017). Contudo, os padrões de distribuição vertical e sazonal da macrofauna bentônica nestes ecossistemas podem variar, especialmente em função dos efeitos do ambiente sobre o ecossistema (Barros \& Rocha-Barreira, 2013; Barros et al., 2017; Edgar, 1990; Jernakoff \& Nielsen, 1998; Nakaoka et al., 2001) e das interações entre espécies da fauna e entre espécies de angiospermas formadoras do prado (Nakaoka, 2005).

No Brasil, os estudos específicos sobre macrofauna bentônica ou grupos bentônicos associados a estes prados têm demonstrado alterações na composição das espécies, em função das variações sazonais ou do ciclo de vida das plantas (Alves \& Araújo, 1999; Barros \& Rocha-Barreira, 2013; Barros et al., 2017; Cavalcante et al., 2014), da distribuição da pradaria em diferentes profundidades (Creed \& Kinupp, 2011), da biomassa e partes aérea e subterrânea destas plantas sobre a distribuição vertical da macrofauna (Barros \& RochaBarreira, 2009/2010; 2013; Barros et al., 2013a; 2017) e, também, da presença das angiospermas marinhas sobre a diversidade da macrofauna bentônica (Corbisier, 1994).

Atualmente, mudanças ambientais de ordem global têm reduzido significativamente as áreas de prados em todo o mundo, afetando seus serviços ambientais (Björk et al., 2008; Duarte, 2002; Green \& Short, 2003; Orth et al., 2006; Waycott et al., 2007; 2009) e a estrutura da macrofauna bentônica associada (Do et al., 2013). Estas alterações já vêm sendo registradas no Brasil, como modificações na abrangência das espécies de angiospermas marinhas (Barros et al., 2013b; Copertino et al., 2016; Gorman et al., 2016), assim como áreas com perda de densidade e biomassa e com pradarias cada vez mais fragmentadas (Short et al., 
2006). No litoral norte do estado de Pernambuco, informações de pescadores registram a perda e fragmentação de área e, também, a queda da produção pesqueira nas últimas décadas (Santos \& Magalhães, em prep.).

Assim, considerando as recentes perdas e fragmentação dos prados de angiospermas marinhas, são considerados urgentes os estudos sobre como as mudanças em sua estrutura impactam a fauna associada (Airoldi et al., 2008). Diante desse cenário, pontos de monitoramento têm sido estabelecidos no litoral Norte de Pernambuco, visando antecipar possíveis alterações na estrutura da fauna, que resultem em perda de espécies de interesse econômico. O presente estudo testou a hipótese de que mesmo em áreas recém-fragmentadas, as áreas vegetadas têm maior diversidade e abundância de macrofauna bentônica.

\section{MATERIAL E MÉTODOS}

\section{Área de estudo}

A praia de Catuama $\left(7^{\circ} 37^{\prime} 40,7^{\prime \prime}\right.$ S e $34^{\circ} 48^{\prime} 19,9^{\prime \prime}$ W) está localizada no litoral norte do estado de Pernambuco, à sudeste do município de Goiana e a $72 \mathrm{~km}$ da capital pernambucana, Recife, nordeste brasileiro. A faixa de praia apresenta 1,5 km de extensão e recebe o encontro do Rio da Barra com o Rio Itapessoca (Figura I). O prado de Halodule wrightii Ascherson foi escolhido para o estudo após terem sido observadas fragmentações, durante trabalhos de campo entre os anos de 2009 e 2010, onde antes eram observados apenas prados contínuos (Santos \& Magalhães, em prep.), sendo o trabalho realizado no início da fragmentação.

Nesta área, o período chuvoso ocorre entre os meses de março e agosto, sendo bem definido por frentes frias vindas do Sul, com uma precipitação pluviométrica média anual de $1720 \mathrm{~mm}$ (Aragão et al., 2004). A temperatura média do ar oscila em torno de $26^{\circ} \mathrm{C}$ e a velocidade média de ventos alísios (predominantes) varia entre 6,1 e 9,3 nós, com ventos oriundos principalmente do Leste, no período de outubro a março; e do sudeste-sul, no período de abril a setembro (Aragão et al., 2004).

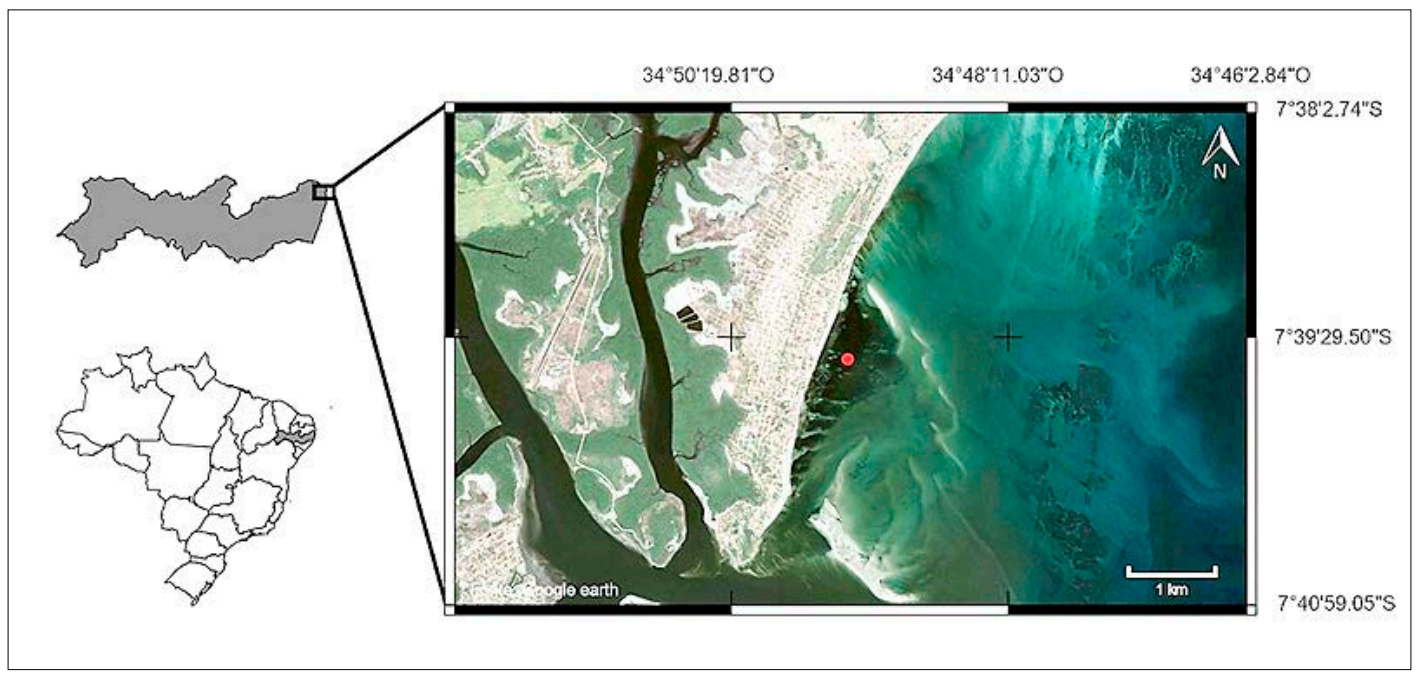

Figura 1 - Localização do local de estudo na Praia de Catuama, no estado de Pernambuco, Nordeste do Brasil. 


\section{Etapa em campo}

Em maio de 2010, foram coletadas 10 amostras dentro das manchas de Halodule wrightii e 10 amostras em áreas sem vegetação, com distância de 1 a 2 metros entre as manchas e áreas não-vegetadas. Para a remoção do substrato contendo as plantas e a macrofauna bentônica associada, utilizou-se um tubo coletor de PVC de $10 \mathrm{~cm}$ de diâmetro, enterrado a $10 \mathrm{~cm}$ de profundidade, conforme metodologia padrão para estudos de fauna associada (Raz-Guzman \& Grizzle, 2001). As amostras foram acondicionadas em sacos plásticos, fixadas com formol salino a $4 \%$ e levadas ao Laboratório de Ecossistemas Aquáticos da Universidade Federal Rural de Pernambuco (LEAqua/UFRPE) para a triagem e identificação dos grupos da macrofauna bentônica.

\section{Etapa em laboratório}

O material coletado foi lavado em água corrente, por meio de uma peneira com malha de $0,5 \mathrm{~mm}$, para a retenção da macrofauna. Nas amostras das áreas vegetadas, as folhas e rizomas de $H$. wrightii foram retiradas para posterior análise. O material retido na malha foi acondicionado em potes plásticos e fixado em formol a $4 \%$, contendo o corante Rosa de Bengala (Holme \& Mclntyre, 1971). A triagem e identificação dos grupos da macrofauna bentônica foram realizadas com auxílio de um microscópio estereoscópico e literatura específica.

O número de hastes de $H$. wrightii foi contado para obtenção da densidade (hastes $\mathrm{m}^{-2}$ ); e as partes aérea (folhas e bainha) e subterrânea (rizoma e raízes) das plantas foram levadas à estufa a $60^{\circ} \mathrm{C}$ por 24 horas, para determinação da biomassa seca $\left(\mathrm{g} \mathrm{ps} \mathrm{m}^{-2}\right)$ (Duarte \& Kirkman, 2001).

\section{Análises estatísticas}

Para os grandes grupos da macrofauna bentônica foram obtidos a abundância total e o número de táxons. Estas variáveis bióticas foram comparadas entre as áreas vegetadas e não vegetadas através da análise não paramétrica de Kruskal-Wallis (ao nível de significância de $\alpha=0,05)$, através do Programa Bioestat, versão 4.0, após testadas a normalidade e homocedasticidade.

Os dados de abundância dos grandes grupos foram previamente transformados pela raiz quadrada, como recomendado por Clarke e Warwick (1994). A partir dos dados de abundância foi construída uma matriz de similaridade usando o Índice de Bray-Curtis e as diferenças espaciais entre as comunidades das áreas vegetadas e não vegetadas foram examinadas através da Ordenação MDS (Escalonamento Multidimensional) (Clarke \& Ainsworth, 1993).

A significância das dissimilaridades entre as estruturas multivariadas das amostras vegetadas e não vegetadas, foi testada através da análise de Similaridade (ANOSIM de uma via). A fim de determinar os táxons mais importantes em termos de contribuição (\%) para a dissimilaridade entre áreas vegetadas e não vegetadas, foi aplicada a análise do percentual de similaridades (SIMPER) (Clarke, 1993). As análises foram realizadas com auxílio do Programa Primer $^{\circledR}$ (Plymouth Routines In Multivariate Ecological Research), versão 6.0. 


\section{RESULTADOS}

Foi capturado um total de 2566 espécimes da macrofauna bentônica, sendo 2493 em áreas vegetadas e 73 em áreas sem vegetação. Foram identificados os filos Annelida, Arthropoda (Crustacea: Amphipoda, Decapoda, Isopoda, Tanaidacea; e Pycnogonida), Cnidaria, Echinodermata (Ophiuroidea), Mollusca e Sipuncula. De todos os táxons identificados, apenas Annelida e Mollusca foram encontrados em ambos os ambientes estudados, enquanto os demais ocorreram exclusivamente associados a Halodule wrightii.

A abundância total e o número de táxons foram significativamente maiores nas áreas vegetadas (Tabela 1). Anelídeos, cnidários e moluscos foram os grupos mais densos nas amostras coletadas em áreas vegetadas, sendo os anelídeos também o grupo mais denso nas áreas não vegetadas (Tabela 2).

O MDS evidenciou alta dissimilaridade $(\delta$ média $=81,34)$ entre as abundâncias dos táxons nas amostras de áreas vegetadas (AP) e não vegetadas (AF) (Figura 2), que foram consideradas significativamente diferentes (ANOSIM de uma via: $R=0,941 ; p=0,01 \%$ ). $O$ grupo que mais contribuiu para a dissimilaridade entre estas áreas foi o filo Annelida $(42,6 \%)$ (Tabela 3$)$.

As áreas vegetadas foram caracterizadas por uma densidade de hastes de Halodule wrightii, variando entre 1590 hastes $\mathrm{m}^{-2} \mathrm{e} 7291$ hastes $\mathrm{m}^{-2}$ com densidade média de $3314 \pm 177,4$ hastes $\mathrm{m}^{-2}$. A biomassa aérea variou entre $52,36 \mathrm{~g} \mathrm{ps} \mathrm{m}^{-2}$ e 165,45 $\mathrm{g} \mathrm{ps} \mathrm{m}^{-2}$, com uma média de $103 \pm 4,1 \mathrm{~g} \mathrm{ps} \mathrm{m}^{-2}$, e a biomassa subterrânea variou entre $117,59 \mathrm{~g} \mathrm{ps} \mathrm{m}^{-2}$ e $415,087 \mathrm{~g}_{\text {ps m}}{ }^{-2}$, com uma média de $237,04 \pm 9,9 \mathrm{~g}_{\mathrm{ps} \mathrm{m}} \mathrm{m}^{2}$.

Tabela 1 - Valores (média \pm Desvio padrão) da Abundância total, número de táxons e resultado da ANOVA para a macrofauna bêntica em áreas de prado de Halodule wrightii e áreas não vegetadas na praia de Catuama, PE, Brasil.

\begin{tabular}{lcccc}
\hline \multirow{2}{*}{ Descritor } & \multicolumn{2}{c}{ Média \pm Desvio-padrão } & \multicolumn{2}{c}{$\begin{array}{c}\text { ANOVA } \\
\text { (Kruskal-Wallis) }\end{array}$} \\
& Vegetada & Não-vegetada & H & p \\
\cline { 2 - 5 } Abundância Total & $6,5 \pm 1,90$ & $1,2 \pm 0,42$ & 15.310 & $<0.001$ \\
Número de Táxons & $27,87 \pm 10,85$ & $2,53 \pm 1,70$ & 13.740 & $<0.001$ \\
\hline
\end{tabular}

Tabela 2 - Média e desvio-padrão da densidade (táxon $\mathrm{m}^{-2}$ ) da macrofauna bentônica em áreas de vegetadas e não vegetadas por Halodule wrightii da praia de Catuama, Pernambuco, Brasil.

\begin{tabular}{lll}
\hline Táxons & $\begin{array}{l}\text { Área vegetada } \\
\text { (média } \pm \text { desvio-padrão) }\end{array}$ & $\begin{array}{l}\text { Área não vegetada } \\
\text { (média } \pm \text { desvio-padrão) }\end{array}$ \\
\cline { 2 - 3 } Annelida & $\begin{array}{l}26276,02 \pm 18505,65 \\
1418,53 \pm 1771,01\end{array}$ & $\begin{array}{l}941,27 \pm 1085,06 \\
\text { Cnidaria }\end{array}$ \\
Mollusca & $212,11 \pm 280,88$ & $26,51 \pm 55,89$ \\
Sipuncula & $13,25 \pm 41,92$ & $*$ \\
Ophiuroidea & $901,49 \pm 787,54$ & $*$ \\
Pycnogonida & $92,80 \pm 109,14$ & $*$ \\
Amphipoda & $3897,65 \pm 2354,35$ & $*$ \\
Decapoda & $92,80 \pm 177,31$ & $*$ \\
Isopoda & $145,83 \pm 245,64$ & $*$ \\
Tanaidacea & $768,92 \pm 858,71$ & $*$ \\
\hline
\end{tabular}

* Ausência de indivíduos 


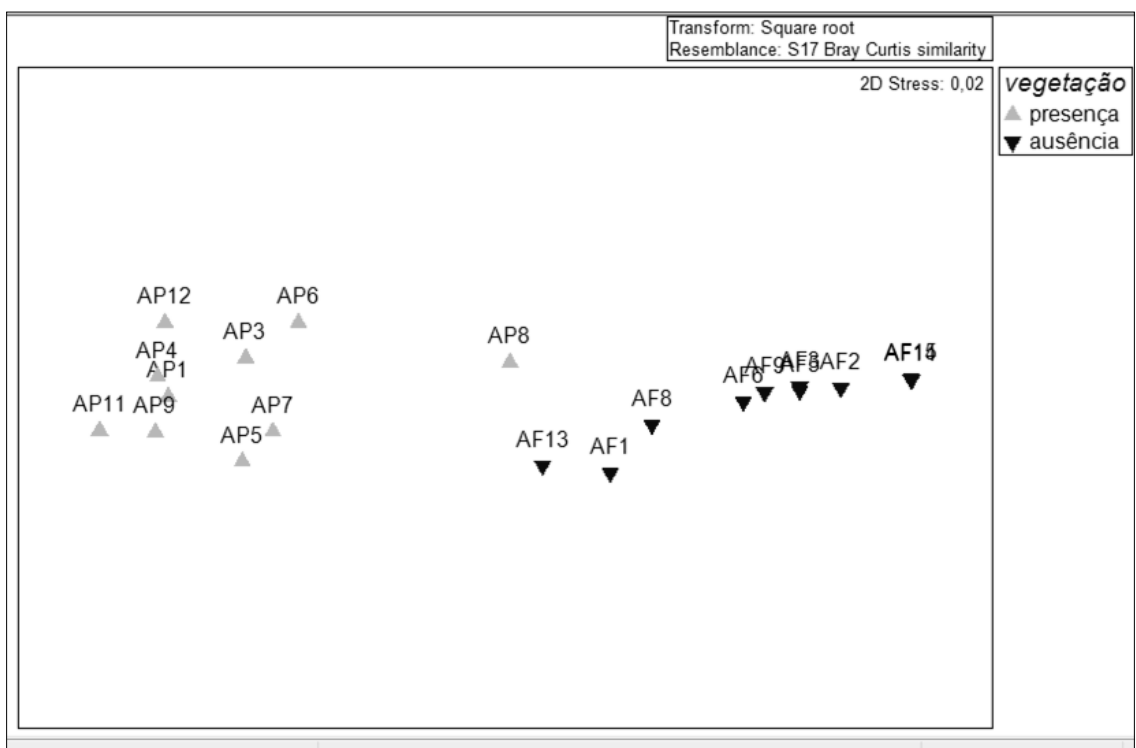

Figura 2 - Gráfico resultante do Escalonamento Multidimensional (MDS) das abundâncias médias dos grupos da macrofauna bentônica associada a áreas vegetadas (presença-AP) e não vegetadas (ausência-AF) por Halodule wrightii, na praia de Catuama, Pernambuco, Brasil.

Tabela 3 - Média de abundância dos grupos da macrofauna bentônica em áreas vegetadas e não vegetadas, e seus percentuais de contribuição para a dissimilaridade média ( $\delta$ média) (Índice de Bray-Curtis) entre as áreas vegetadas e não vegetadas por Halodule wrightii na praia de Catuama, Pernambuco, Brasil.

\begin{tabular}{lrrr}
\hline \multirow{1}{*}{ Táxons } & \multicolumn{2}{c}{ Abundância Média } & \multirow{2}{*}{ Contribuição (\%) } \\
\hline Annelida & Área vegetada & Área não-vegetada & 42,62 \\
Amphipoda & 198,2 & 7,1 & 20,66 \\
Cnidaria & 29,4 & 0,0 & 10,63 \\
Ophiuroidea & 10,7 & 0,0 & 8,43 \\
Tanaidacea & 6,8 & 0,0 & 7,26 \\
Mollusca & 5,8 & 0,0 & 3,56 \\
Isopoda & 1,6 & 0,2 & 2,76 \\
Decapoda & 1,1 & 0,0 & 1,44 \\
Pycnogonida & 0,7 & 0,0 & 2,30 \\
Sipuncula & 0,7 & 0,0 & 0,33 \\
\hline
\end{tabular}

\section{DISCUSSÃO}

Trabalhos sobre fragmentação realizados em ecossistemas de angiospermas marinhas não fazem, em geral, distinção entre fragmentação e perda de habitat. Contudo, os autores utilizam o termo fragmentação para indicar diminuição na proximidade e conectividade entre manchas, ou o aumento do isolamento desses prados (Bostrom et al., 2006). Essa fragmentação pode causar efeito de borda sobre a macrofauna bentônica, especialmente por sua localização em zonas rasas costeiras, o que as torna ainda mais vulneráveis aos efeitos antrópicos, acelerando os processos de fragmentação (Waycott et., 2009). Ainda há controvérsias quanto aos efeitos de borda para os diversos grupos da fauna associada a 
prados de angiospermas marinhas, se por um lado parecem não ter efeito significativo para a infauna em curtas distâncias (1-15m) (Summerson \& Peterson 1984; Bell et al., 2001), estudos já demonstraram efeitos positivos (Vonk, et al., 2010; Arponen \& Bostrom, 2012), enquanto outros verificaram que a partir de 1 metro já há efeito negativo, relacionado ao aumento da exposição à predação, em especial para a infauna móvel (Tanner 2005). A capacidade de dispersão dos grupos tem influência direta sobre esses efeitos (Virnstein \& Curran, 1986; Robbins \& Bell, 1994), mas também tem sido sugerido que a proximidade de outros habitats pode influenciar a fauna (Ollivier et al., 2015).

As manchas de pradaria funcionam como filtros de ondas e correntes dispersos pelo substrato, capturando grande número de larvas e pequenos animais (Bologna \& Heck-Jr, 2002; Sánchez-Jerez et al., 1999), podendo manter as espécies-chave até o restabelecimento do ecossistema. No entanto, apesar das manchas da pradaria de Catuama terem apresentado os grupos da macrofauna bentônica característicos para este tipo de ambiente (Barnes et al., 2017; Barros \& Rocha-Barreira, 2009/2010; Casares \& Creed, 2008; Corbisier 1994; Hemminga \& Duarte 2000; Nakaoka \& Toyohara 2000; Stoner, 1980), este estudo confirmou a hipótese de que as áreas onde ocorreu perda de cobertura vegetal recente, foram significativamente menos habitadas pela macrofauna bentônica, com provável impacto também significativo sobre a produtividade local. O mesmo foi verificado em áreas recém fragmentadas por motores de barcos, onde houve redução significativa na abundância de moluscos e camarões (Uhrin \& Holmquist, 2003).

Efeito similar também foi observado numa pradaria de Zostera noltii Hornemann com perda de área, onde os autores registraram uma queda significativa na macrofauna bentônica nas áreas com perda de cobertura vegetal, especialmente considerando os grupos da epifauna (Do et al., 2013). Outros estudos que compararam áreas vegetadas e não vegetadas também registraram maior abundância nas áreas com plantas (Do et al., 2011; Orth et al., 1984; Sánchez-Jerez et al., 1999; Terrados \& Borum, 2004; Virnstein et al., 1983; Zieman \& Zieman, 1989), diferenciando quanto aos grupos faunísticos mais destacados, o que pode estar relacionado à espécie de planta e outras particularidades ambientais de cada local.

O destaque do grupo Annelida, especialmente os poliquetas, também tem sido evidenciado em outras pradarias (Gambi et al., 1998; Omena \& Creed, 2004; Somaschini et al., 1994; Virnstein, 1987; Stoner,1980; Hutchings, 1993; Corbisier, 1994). Alguns autores sugerem que este grupo seja mais fortemente influenciado pelo sedimento que pelas plantas, em função do hábito (Froján et al., 2005; Corbisier, 1994) ou do sucesso reprodutivo neste tipo de habitat (Bell., et al., 2001). No entanto, outros autores afirmam que espécies deste grupo podem estar diretamente relacionadas à biomassa das plantas (Gambi et al., 1998; Omena \& Creed, 2004; Arana \& Díaz, 2006) e encontrar maior proteção e alimento em áreas vegetadas, mesmo quando as espécies de plantas apresentam menor porte (Abbiatti et al., 1991; Gambi et al., 1998; Omena \& Creed, 2004; Somaschini et al., 1994).

No presente estudo, o grupo dos moluscos também foi observado, tanto nas áreas vegetadas quanto não vegetadas. Embora tenha sido registrada uma baixa abundância, os moluscos compõem um grupo bastante comum nestes habitats e quase sempre estão diretamente relacionados às variações espaciais e sazonais das pradarias (Alves \& Araújo, 1999; Barros et al., 2013a; Barros \& Rocha-Barreira, 2013; Creed \& Kinupp, 2011; Toyohara \& Nakaoka, 1999) e à presença das plantas, quando comparados a ambientes desprovidos de vegetação (De Metrio et al., 1980; Rueda et al., 2009), com estudos confirmando o efeito de borda em ambientes com vegetação mais fechada (Vonk, et. Al., 2010). 
Outro grupo bem representado no presente estudo foi o subfilo Crustacea, que tem sido apontado como um grupo que tem papel significativo na produção secundária dos prados de angiospermas marinhas (Hemminga \& Duarte, 2000; Nakaoka, 2005; Rainer \& Unsworth, 1991). Os crustáceos são atraídos pela complexidade e estabilidade encontrada nos microambientes formados pelas pradarias (Zupo, 1994). Sua diversidade é regulada principalmente pela densidade aérea da planta, presença de epífitas e sazonalidade das plantas hospedeiras (Stoner, 1980; Gore et al., 1981; Lewis, 1984; Zieman \& Zieman, 1989; Barros \& Rocha-Barreira, 2009/2010; Barros et al., 2017). No presente estudo, as diversas ordens identificadas foram encontradas exclusivamente nas áreas vegetadas, confirmando a relação direta deste grupo com as plantas, como observado por outros autores (SánchezJerez et al., 1999; Lewis, 1984; Stoner, 1980; Zieman \& Zieman, 1989).

Morfologicamente, embora apresentando perdas de área, as médias de tamanho e densidade das plantas, assim como a biomassa, ainda superam os valores encontrados em outras pradarias do Brasil (Barros \& Rocha-Barreira, 2014; Creed, 1999; França et al., 2014; Magalhães et al., 1997; Magalhães et al., 2003; Oliveira et al., 1997). Além disso, o número significativamente maior de macrofauna associada às áreas ainda vegetadas reforça a importância desse ecossistema para a produtividade na região costeira de Catuama, que, mesmo sem a identificação dos grupos ao nível de espécies para uma análise funcional dessa fauna, os resultados demonstraram o quanto pode ser perdido devido à fragmentação de área do prado.

Assim como em outros ecossistemas terrestres ou aquáticos, o efeito da fragmentação em prados de angiospermas marinhas leva a uma perda de diversidade, diversidade genética e dispersão de sementes (Livernois et al., 2017). A uma taxa de perda que varia entre 2 a $5 \%$ ao ano (Orth et al., 2006), os prados de angiospermas marinhas têm desaparecido rapidamente da maior parte das regiões costeiras, com estimativa global de pelo menos 30\% de áreas perdidas (Waycott et al., 2009). Ainda assim, as pradarias da praia de Catuama ainda demonstram sua fundamental importância para a produtividade local. No entanto, a drástica redução na abundância total e número de táxons da macrofauna bentônica nas áreas com perda de pradaria reforça os alertas para os efeitos da destruição desses ambientes não só para a economia, como também para o equilíbrio de toda a zona costeira.

Agradecimentos - Os autores agradecem à ecóloga e doutoranda em Ciências Marinhas Tropicais, Ravena Alves Nogueira, pela elaboração do mapa da área de estudo.

\section{REFERÊNCIAS}

Abbiatti, M.; Castelli, A.; Giangrande, A. \& Lardicci, C. Distribution of polychaetes on hard substrates of the Midlittoral-Infralittoral transition zone, Western Mediterranean. Ophelia (Suppl.), v. 5, p. 421-432, 1991.

Airoldi, L.; Balata, D. \& Beck, M. W. The Gray Zone: Relationships between habitat loss and marine diversity and their applications in conservation. J. Exp. Mar. Biol. Ecol., v. 366, n. 1-2, p. 8-15, 2008.

Alves, M.S. \& Araújo, M.J.G. Moluscos associados ao fital Halodule wrightii Ascherson na Ilha de Itamaracá-PE. Trab. Oceanog., v. 27, n. 1, p. 91-99, 1999.

Aragão, J. O. R. A influência dos Oceanos Pacífico e Atlântico na dinâmica do tempo e do clima do Nordeste do Brasil, p. 287-317, in Eskinazi-Leça, E.; Neumann-Leitão, S. \& Costa, M. F. (orgs.). Oceanografia - um cenário tropical, Bagaço, 761p., Recife, 2004. 
Arana, I. L. \& Díaz, O. D. Polychaeta (annelida) associated whit Thalassia testudinum in the Northeastern coastal waters of Venezuela. Rev. Biol. Trop., v. 54, n. 3, p. 971-978, 2006.

Barnes, R. S. K. \& Barnes, M. K. S. Shore height and differentials between macrobenthic assemblages in vegetated and unvegetated areas of an intertidal sandflat. Estuar. Coast. Shelf Sci., v. 106, p. 112-120, 2012.

Barnes, R. S. K. Patterns of benthic invertebrate biodiversity in intertidal seagrass in Moreton Bay, Queensland. Regional Studies in Marine Science, v. 15, p.17-25, 2017.

Barros, K. V. S. \& Rocha-Barreira, C. A. Caracterização da dinâmica espaço-temporal da macrofauna bentônica em um banco de Halodule wrightii Ascherson (Cymodoceaceae) por meio de estratificação. Rev. Nord. Zool., v. 4, n. 1, p. 73-81, 2009/2010.

Barros, K. V.S. \& Rocha-Barreira, C. A. Environmental influences on Halodule wrightii Ascherson meadow in Northeastern Brazil. Braz. J. Aquat. Sci. Technol., v. 18, n. 2, p. 31-41, 2014.

Barros, K. V.S. \& Rocha-Barreira, C. A. Responses of the molluscan fauna to environmental variations in a Halodule wrightii Ascherson ecosystem from Northeastern Brazil. An. Acad. Bras. Ciênc., v. 85, n. 4, p. 187-200, 2013.

Barros, K. V.S.; Jardim, J. \& Rocha-Barreira, C. A. Ecological observations on Polyplacophora in a Halodule wrightii Ascherson meadow and new records for Northeast and Brazilian coast. Rev. Nord. Zool., v. 7, n. 1, p. 27-40, 2013 a.

Barros, K. V. S.; Rocha-Barreira, C. A. \& Magalhães, K. M. Ecology of Brazilian seagrasses: Is our current knowledge sufficient to make sound decisions about mitigating the effects of climate change? Iheringia Sér. Bot., v. 68, n. 1, p.155-170, 2013b.

Barros, K. V. S.; Souza-Filho, J. F.; Paiva, R. J. C.; Araújo-Silva, C. L.; Almeida, A. O. \& Rocha-Barreira, C. A. Seasonal variation of the crustacean fauna in the belowground and aboveground strata in a Halodule wrightii meadow of northeastern Brazil. Iheringia Sér Zool., v. 107, n. e2017048, p. 1-7, 2017.

Bell,S.S.; Brooks, R.A.; Robbins, B.D.; Fonseca, M.S.; Hall, M.O. Faunal response to fragmentation in seagrass habitats: implications for seagrass conservation. Biol. Conserv., v. 100, p. 115-123, 2001.

Björk, M.; Short, F.; McLeod, E. \& Beer, S. Managing seagrasses for resilience to climate change. World Conservation Union Global Marine Programme, Gland,55 p., Switzerland, 2008.

Bologna, P.A.X.; Heck-Jr, K.L. Impact of Habitat Edges on Density and Secondary Production of Seagrass-associated Fauna. Estuaries, v. 25, p. 1033-1044, 2002.

Casares, F.A. \& Creed, J.C. Do Small Seagrasses Enhance Density, Richness, and Diversity of Macrofauna? J Coastal Res., v. 24, n. 3, p. 790-797, 2008.

Cavalcante, L.L.; Amorim, L.A.; Costa, F.N.; Rocha-Barreira, C.A. \& Barros, K.V. S. Variações no prado de Halodule wrightii Ascherson e macrofauna associada na praia da Pedra Rachada, Paracuru, Ceará - Brasil. Rev. Educ. Cient. Cult., v. 1, n.2, p. 1-9, 2014.

Clarke, K.R. \& Warwick, R.M. Similarity-based testing for community patter: the 2-way layout with no replication. Mar. Biol., v. 18, p. 167-176, 1994.

Clarke, K.R. \& Ainsworth, M. A method of linking multivariate community structure to environmental variables. Mar. Ecol. Prog. Ser., v. 92, p. 205-219, 1993.

Clarke, K.R. Non-parametric multivariate analyses of changes in community structure. Australian J. Ecology., v.18, p.117-143.1993. 
Copertino, M.S.; Creed, J.C.; Lanari, M.O.; Magalhães, K.; Barros, K.; Lana, P.C.; Sordo, L. \& Horta, P.A. Seagrass and submerged aquatic vegetation (VAS) habitats off the coast of Brazil: state of knowledge, conservation and main threats. Braz. J. Oceanogr., v. 64, n. sp2, p. 53-80, 2016.

Corbisier, T.N., Macrozoobentos da Praia do Codó (Ubatuba,SP) e a presença de Halodule wrightii. Bolm. inst. oceanogr., v. 42, p. 99-111, 1994.

Costanza, R.; D’arge, R.; Groot, R.; Farberk, S.; Grasso, M.; Hannon, B.; Limburg, K.; Naeem, S.; O'neill, R.V.; Paruelo, J.; Raskin, R.G.; Suttonkk, P. \& Van Den Belt, M. The value of the world's ecosystem services and natural capital. Nature, v. 387, p. 253-260, 1997.

Creed, J.C. \& Kinupp, M. Small scale change in mollusk diversity along a depth gradient in a seagrass bed off Cabo Frio, (Southeast Brazil). Braz. J. Oceanogr., v. 59, n. 3, p. 267-276, 2011.

Creed, J.C. Distribution, seasonal abundance and shoot size of the seagrass Halodule wrightii near its southern limit at Rio de Janeiro state, Brazil. Aquat. Bot. v. 65, p. 47-58, 1999.

De Metrio, G.; Bello, G.; Vaccarella, R. \& Terio, E. Malacofauna di "mattes" morti di Posidonia. Atti Accad. Peloritana Pericolanti Cl. Sci. Fis. Mat. Natur., v. 26, p. 3-8, 1980.

Do, V.T.; Blanchet, H.; Montaudouin, X. \& Lavesque, N. Limited Consequences of Seagrass Decline on Benthic Macrofauna and Associated Biotic Indicators. Estuaries Coast., v. 36, p. 795-807, 2013.

Do, V.T.; Montaudouin, X.; Lavesque, N.; Blanchet, H. \& Guyard, H. Seagrass colonization: knock-on effects on zoo- benthic community, populations and individual health. Estuar. Coast. Shelf Sci., v. 95, p. 458-469, 2011.

Duarte, C.M. The future of seagrass meadows. Environ. Conserv., v. 29, n. 2, p. 192-206, 2002.

Duarte, C.M. \& Kirkman, H. Methods for the Measurement of Seagrass Abundance and Depth Distribution, p. 141-153, in Short, F.T. \& Coles, R.G. (eds.), Global Seagrass Research Methods. Elsevier, 463p., New York, 2001.

França, C.R.C.; Pitanga, M.E.; Alves, M.D.O.; Araújo, M.E.; Silva, S.L. \& Magalhães, K.M. Morfologia foliar e densidade de hastes de Halodule wrightii (Cymodoceaceae), no litoral de Alagoas, Brasil. Trop. Oceanogr., v. 42, n. esp., p. 58-67, 2014.

Froján, C.R.S.; Hawkins, L.E.; Aryuthaka, C.; Nimsantijaroen, S.; Kendall, M.A. \& Paterson, G.L.J. Patterns of polychaete communities in tropical sedimentary habitats: a case study in south-western Thailand. Raffles Bull. Zool., v. 53, n. 1, p. 1-11, 2005.

Gambi M.C.; Conti G. \& Bremec C.S. Polychaete distribution, diversity and seasonality related to seagrass cover in shallow bottoms of the Tyrrenian Sea (Italy). Sci. Mar., p. 62: $1-2,1998$.

Gore, R.H.; Gallaher, E.E.; Scotto, L.E. \& Wilson, K.A. Studies on decapod crustacea from theIndian River Region of Florida: XI. Community composition, structure, biomass and species-areal relationships of seagrass and drift algae-associated macrocrustaceans. Estuar. Coast. Shelf Sci., v.12, n. 4, p. 485-508, 1981.

Gorman, D.; Turra, A.; Bergstrom, E. \& Horta, P.A. Population expansion of a tropical seagrass (Halophila decipiens) in the southwest Atlantic (Brazil). Aquat. Bot., v. 132, p. 30-36, 2016.

Green, E.P. \& Short, F.T. World Atlas of Seagrasses. University of California Press, 298p., Los Angeles, 2003. 
Heck, K.L. \& Orth, R.J. Structural components of eelgrass (Zostera marina) meadows in the lower Chesapeake Bay-Decapod crustacea. Estuar Coast., v. 3, n. 4, p. 289-295, 1980.

Heidi Arponen, H. \& Bostrom, C. Responses of mobile epifauna to small-scale seagrass patchiness: is fragmentation important? Hydrobiologia. V. 680, p. 1-10. 2012.

Hemminga, M.A. \& Duarte, C.M. Seagrass Ecology. Cambridge University Press, 298p., Cambridge, 2000.

Holme, N.A. \& McIntyre, A.D. Methods for the study of marine benthos, $1^{\text {st }}$ ed., Blackwell Scientific Publication, 334 p., Oxford, 1971.

Hughes, R.G; Rubies, A.G. \& Gili, J.M. The growth and degeneration of the hydroid Sertularia perpusilla, an obligate epiphyte of leaves of the seagrass Posidonia oceanica. Hydrobiologia, v. 216/217, p. 211-214, 1991.

Hutchings, P.A.; Ward, T.J. \& Waterhouse, J.H. Infauna of Marine Sediments and Seagrass Beds Of Upper Spencer Gulf Near Port Pirie, South Australia. Summ. Trans. Royal Soc., v. 117, n. 1, p. 1-15, 1993.

Jernakoff, P. \& Nielsen, J. Plant-animal associations in two species of seagrasses in Western Australia. Aquat. Bot., v. 60, p. 359-376, 1998.

Lewis, F. G. Distribution of macrobenthic crustaceans associated with Thalassia, Halodule and bare sand substrata. Mar. Ecol. Progr. Ser., v. 19, p. 101-113, 1984.

Magalhães, K.M.; Eskinazi-Leça, E.; Moura Júnior, A.M. Morfometria e Biomassa da Fanerógama Marinha Halodule wrightii Ascherson no Litoral Norte de Pernambuco.Trab. Oceanogr., v. 25, p. 83-92. 1997.

Nakaoka, M. \& Toyohara, T. Effects of seagrass patch structure on the mobile epifaunal community in a subtidal seagrass meadow in Thailand. Benthos Res., v. 55, p. 53-61, 2000.

Nakaoka, M. Plant-animal interactions in seagrass beds: ongoing and future challenges for understanding population and community dynamics. Popul. Ecol., v. 47, n. 3, p. 167177, 2005.

Nakaoka, M.; Toyohara, T. \& Matsumasa, M. Seasonal and between-substrate variation community in a multiespecific seagrass bed of Otsuchi Bay, Japan. Mar. Ecol., v. 22, n. 4, p. 379-395, 2001.

Oliveira, E.C., Corbisier, T.N., de Eston, V.R., \& Ambrósio Jr, O. Phenology of a seagrass (Halodule wrightii) bed on the southeast coast of Brazil. Aquat. Bot., Amsterdam, v. 56, n. 1, p. 25-33, 1997.

Ollivier, A,B.; Bramwell; , N. A.; Hammill, E.; Foster-Thorpe, C.; Booth, D.J. Are the effects of adjacent habitat type on seagrass gastropod communities being masked by previous focus on habitat dyads? Aust. J. Zool., v. 63, p. 357-363, 2015.

Omena, E. \& Creed, J.C. Polychaete Fauna of Seagrass Beds (Halodule wrightii Ascherson) Along the Coast of Rio de Janeiro (Southeast Brazil). Mar. Ecol., v. 25, n. 4, 273-288, 2004.

Orth, R.J.; Heck-Jr, K.L. \& Van Montfrans, J. Faunal communities in seagrass beds: a review of the influence of the plant structure and prey characters on predator: prey relationships. Estuaries, v. 7, n. 4, p. 339-350, 1984.

Rainer, F. \& Unsworth, P. Ecology and production of Nebalia sp. (Crustacea: Leptostraca) in a shallow-water seagrass community. Austr. J. Mar. Freshwater Res., v. 42, p. 53-68, 1991. 
Raz-Guzman, A. \& Grizzle, R.E. Techniques for quantitative sampling of infauna and small epifauna in seagrass. p. 237-253, in Short, F.T. \& Coles, R.G. (eds.), Global Seagrass Research Methods. Elsevier, 463p., New York, 2001.

Robbins, B.D. \& Bell, S.S. Seagrass landscapes: a terrestrial approach to the marine subtidal environment. Trends Ecol. Evol., v. 9, p. 301-304. 1994.

Rueda, J.L.; Gofas, S.; Urra, J. \& Salas, C. A highly diverse molluscan assemblage associated with eelgrass beds (Zostera marina L.) in the Alboran Sea: Micro-habitat preference, feeding guilds and biogeographical distribution. Sci. Mar., v. 73, n. 4, p. 679-700, 2009.

Sánchez-Jerez, P.; Cebrián, C.B. \& Esplá, A.A.R. Comparison of the epifauna spatial distribution in Posidonia oceanica, Cymodocea nodosa and unvegetated bottoms: Importance of meadow edges. Acta Oecol., v. 20, n. 4, p. 391-405, 1999.

Short, F.T.; Koch, E.W.; Creed, J.C.; Magalhães, K.M.; Fernandez, E. \& Gaeckle, J.L. SeagrassNet monitoring across the Americas: case studies of seagrass decline. Mar. Ecol., v. 27, p. 277-289, 2006.

Somaschini, A.; Gravina, M.F. \& Ardizzone, G.D. Polychaete depth distribution in a Posidonia oceanica bed (rhizome and matte strata) and neighbouring soft and hard bottoms. Mar. Ecol., v. 15, p. 133-151, 1994.

Stoner, A.W. The Role of Seagrass Biomass in The Organization of Benthic Macrofaunal Assemblages. Bull. Mar. Sci., v. 30, n.3, p. 537-551, 1980.

Tanner, J.E. Edge effects on fauna in fragmented seagrass meadows. Aust. Ecol. V. 30, p. $210-$ 2018. 2005.

Terrados, J. \& Borum, J. Why are seagrasses important? - Goods and services provided by seagrass meadows, p.8-10, in Borum, J.; Duarte, C. M.; Krause-Jensen, D. \& Greve, T.M. (eds.), European Seagrasses: an introduction to monitoring and management, Monitoring and Management of European Seagrass Beds, 88p., Denmark, 2004.

Toyohara, T. \& Nakaoka, M. Population dynamics and reproductive traits of phytal gastropods in seagrass bed in Otsuchi Bay, northeastern Japan. Mar. Ecol., v. 20, n. 3-4, p. 273-289, 1999.

Uhrin, V. \& Holmquist, J.G. Effects of propeller scarring on macrofaunal use of the seagrass Thalassia testudinum. Mar Ecol Prog Ser. v. 250, p.61-70. 2003.

Virnstein, R. \& Curran, M. Colonization of artificial seagrass versus time and distance from source. Marine Ecology Progress Series 29: 279-288. 1986.

Virnstein, R. W. Seagrass-associated Invertebrate Communities of the Southeasthern U.S.A.: A Review, p.89-116, in Durako, M. J.; Philips, R. C. \& Lewis, R.R. (eds.), Proceedings of the Symposium on Subtropical - Tropical Seagrass of the Southeastern United States, Florida Marine Research Publications, Florida, 1987.

Vonk, J.A.; Christianen, M.J.A. Stapel, J. Abundance, edge effect, and seasonality of fauna in mixed-species seagrass meadows in southwest Sulawesi, Indonesia. Mar. Biol. Res. V. 6, p. 282-291, 2010.

Waycott, M.; Collier, C.; Mcmahon, K.; Ralph, P.; Mckenzie, L.; Udy, J. \& Grech, A. Vulnerability of seagrasses in the Great Barrier Reef to climate change, p. 193-235, in Johnson, J. E. \& Marshall, P. A. (eds.), Climate Change and the Great Barrier Reef: a vulnerability 
assessment. Great Barrier Reef Marine Park Authority and Australian Greenhouse Office, Townsville, 2007.

Waycott, M.; Duarte, M.C.; Carruthers, J.B.T.; Orthd,J.R.; Dennisonc, W.C.; Olyarnike, S.; Calladinea, A.; Fourqureanf, J.W.;HeckJr, K.L.;Hughese, A.R.;Kendricki, G.A.;Kenworthyj, W.J.; Short, F.T. \& Williams, L.S. Accelerating Loss Of Seagrasses Across The Globe Threatens Coastal Ecosystems. Pnas, v. 106, n. 30, p. 12377-12381, 2009.

Zieman, J.C. \& Zieman, R.T. The ecology of the seagrass meadows of the west coast of Florida: a community profile. U.S. Department of the Interior Fish and Wildlife Service Research and Development, Biological Report, Washington, D. C., v. 85, n. 7.25, 155p., 1989.

Zupo, V. Strategies of sexual inversion in Hippolyte inermis Leach (Crustacea, Decapoda) from a Mediterranean seagrass meadow. J. Exp. Mar. Biol. Ecol., v.178, p. 131-145, 1994. 